\title{
Impact of structured education on Diabetes management during Ramadan
}

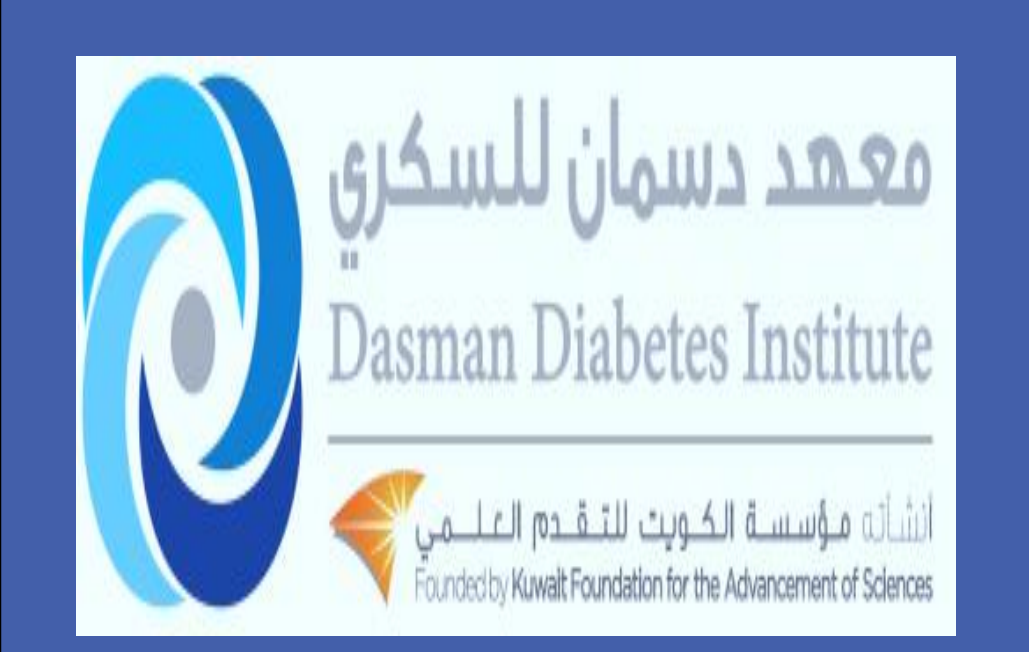

\author{
M.H. Jalali' ${ }^{1}$, F. Mahmoud ${ }^{2}$, N. Othman², N. Al Matrouk ${ }^{3}$, Monira Al Arouj ${ }^{4}$, A. Bennakhi ${ }^{4}$. \\ 1 Nursing Dept., Dasman Diabetes Institute, Kuwait; ${ }^{2}$ Education Dept., Dasman Diabetes Institute, \\ Kuwait; ${ }^{3}$ Nutrition Dept. , Dasman Diabetes Institute, Kuwait; ${ }^{4}$ Diabetes Consultant , Dasman \\ Diabetes Institute, Kuwait.

\section{Introduction}

Fasting during the holy month of Ramadan is an obligatory duty for all healthy adult Muslims. In Islam sick people who suffer from chronic diseases are exempted from fasting. Many Muslims with diabetes, despite the religious and medical advice, prefe to fast exposing themselves to different complications. This was shown in EPIDIAR Study ${ }^{1}$ which revealed that complications such as hypo and hyperglycaemia can be exacerbated by 3-4 folds in the people with diabetes fasting Ramadan. On the other hand diabetic patients mostly have low confidence to manage their glycemic imbalance during Ramadan due to their knowledge deficit. Therefore structured education \& support can be of benefit to minimize these risks and make fasting as safely as possible.

\section{$\underline{\text { Aim }}$}

The aim of this study is to:

Evaluated the impact of implementing a structured education program in preventing diabetes associate complications of fasting.

\section{Method}

$>$ A customized version of MADAR ${ }^{2}$ (Measured Approach for Diabetes and Ramadan) has been adopted, adapted and implemented for this purpose. Educators, nutritionists, nurses and physicians are involved in this program.

$>$ The course is a one day of 3 hours duration. It consists of two parts; basic diabetes and nutritional concepts.

- Ramadan -focused structural diabetes education program targeting people (male / female) who wish to fast during Ramadan conducted one month before starting of Ramadan 2014-2015 and 2016 in weekly basis in Dasman Diabetes Institute.

\section{Assessment tools}

A survey which was adapted from MADAR was used to assess the impact of the education program and collecting data consist of:

$>$ Patient's Demographic information.

- Basic information about current diabetes status (Type of Diabetes, Current treatment, history of last Ramadan hypoglycemic events, Basic diabetes management knowledge assessment.

$>$ Confidence scale assessment table with confidence rate from $0 \%$ to $100 \%$ pre education session and post education session

Comparison between the Latest HbA1c report before and after Ramadan for glycemic control evaluation.

- All participant asked to fill a pre -session survey, then two separate educational power points presented to the attendance by diabetes educator and nutritionist, at the end of each section all participants have been given enough time to raise their questions and to fill the post session survey.

- In order to report any possible hypoglycemic events during fasting period in Ramadan a direct phone number for all participants has been provided

\section{Results}

215 patients, 118 male and 97 female, were invited and accepted to participate in the program. Only $18 / 215$ were T1DM, the remaining 197 were T2DM. The mean age of the participants was 56.1 year.

All patient fasted for 30 days

$>$ There was a significant improvement of confidence rate to manage diabetes outcomes during fasting. (improved from $65.05 \%$ before education session to 94.1\% after education sessions.)

There was $46.9 \%$ reduction in hypoglycemic events and an overall improvement in glycemic control by $1.1 \%$ of HbA1c.

$>$ Two patients needed emergency visits or hospitalization.

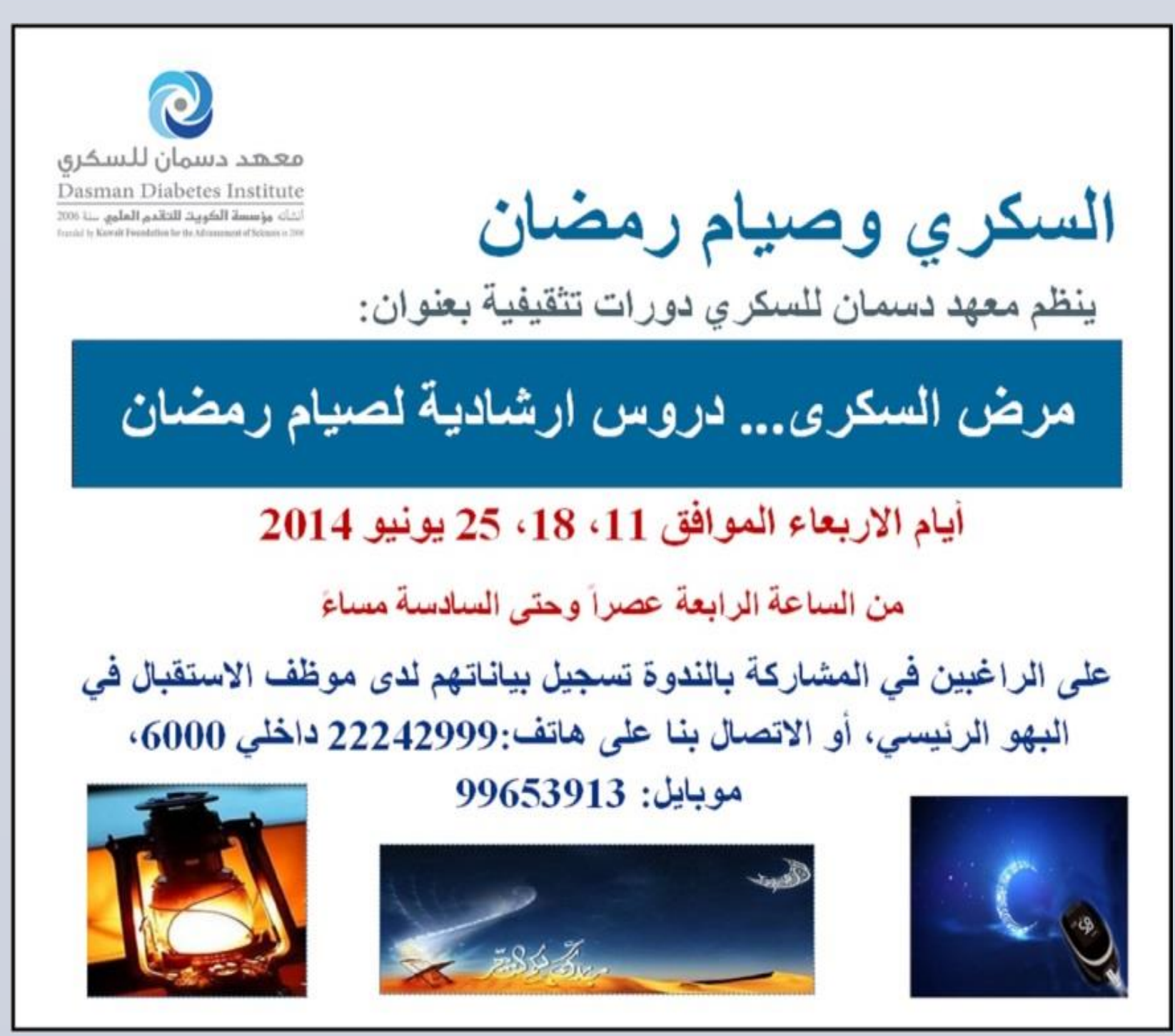

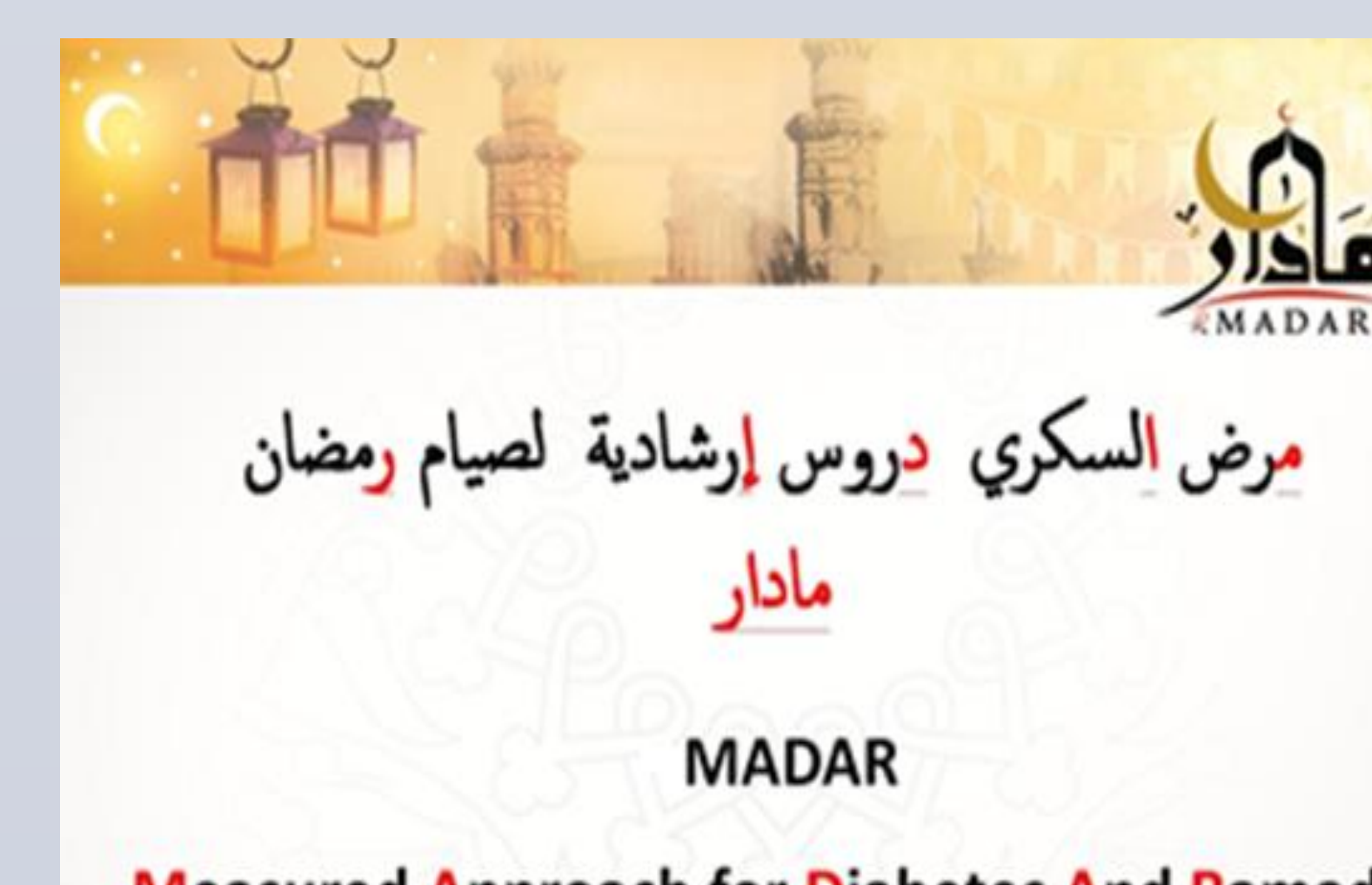

Measured Approach for Diabetes And Ramada Dr. Mohamed Hassanein

Program supported by DESG \& GGSD

\section{RESULTS}

\begin{tabular}{|l|c|c|c|c|}
\hline \multicolumn{5}{|c|}{ RESULTS } \\
\hline & 2014 & 2015 & 2016 & Total \\
\hline Previous Education & $4 / 50$ & $08 / 60$ & $32 / 105$ & $44 / 215$ \\
\hline
\end{tabular}

\section{Mean SMBG}

Compliance

Treatment Adjustment Compliance

Hospitalization

Hba Ic Improvement

$98 \%$

$100 \%$

$100 \%$

$99 \%$

\section{Mean Confidence Rate:}

\section{Before}

Education

After

Education

$68 \%$

$100 \%$

$80.2 \%$

$84.3 \%$

\section{0}

$09 \%$

$32 / 50$

0

$1.3 \%$

$39 / 60$

2

2

$1.1 \%$

$36 / 105$

$1.1 \%$
$71 / 100$

$100 \%$

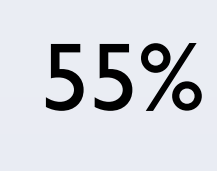

$68.16 \%$

$72 \%$

$61.58 \%$

$88 \%$

$99.33 \%$

$95 \%$

$93.66 \%$

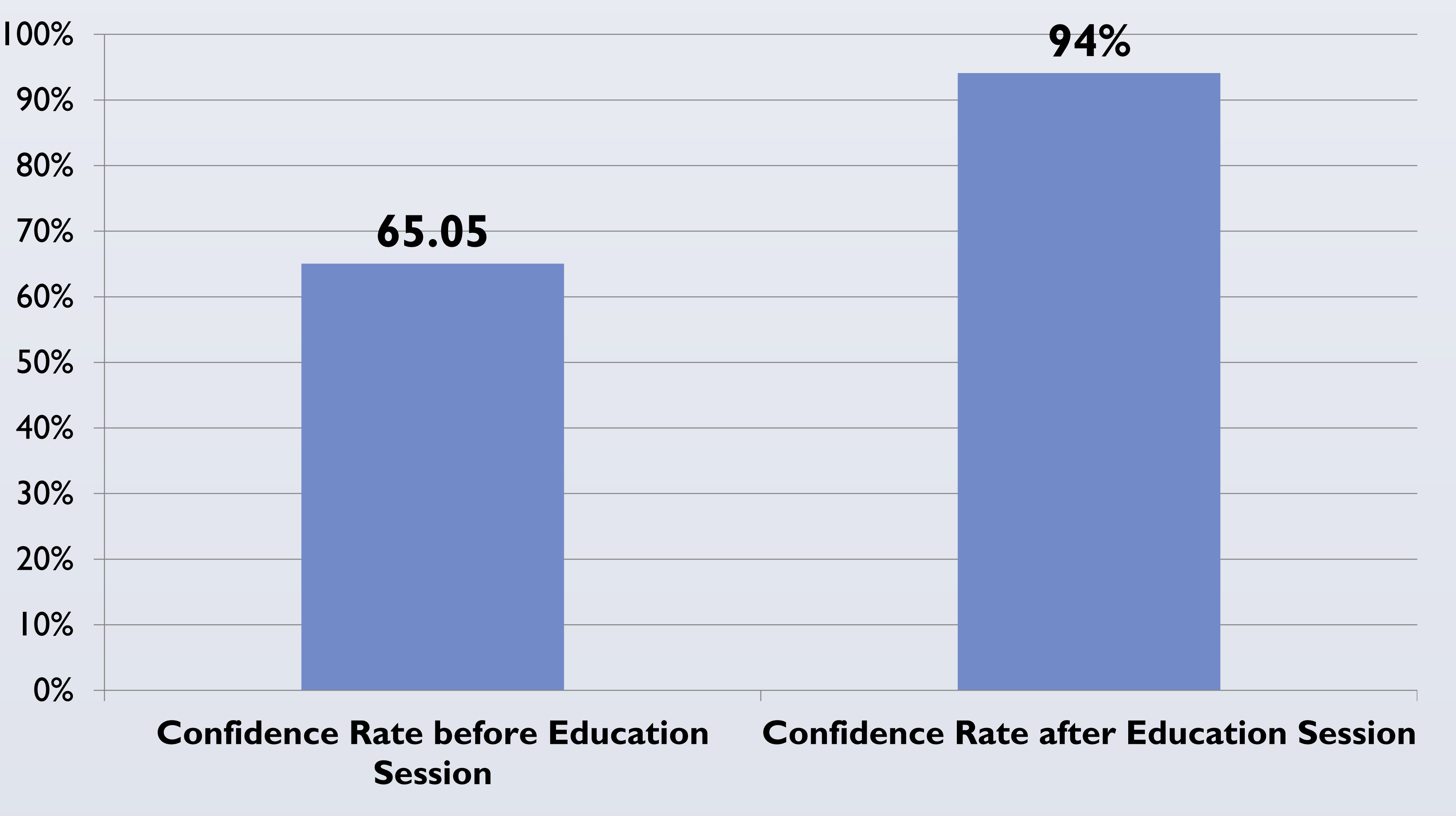

Hypoglycemia Reduction Rate

\section{4}

2015

2016

TOTAL

Hypoglycemia
Incidence
During

Ramadan

\section{Before} Education

$38 / 50$

$46 / 50$

$71 / 105$

$155 / 215$

$72.09 \%$

\begin{tabular}{c|c|c|c|c|}
$\begin{array}{c}\text { After } \\
\text { Education }\end{array}$ & $16 / 50$ & $07 / 60$ & $31 / 105$ & $54 / 215$ \\
\hline & & & $25.1 \%$ \\
\hline
\end{tabular}

\section{Conclusion \& Recommendation}

$>$ Ramadan-focused structural diabetes education program can empower patients to change their lifestyle during Ramadan and it reduces the risks associated with fasting in people with diabetes.

$>$ We can advise this successful structured education program to other health care providers in diabetes -clinics in Kuwait and plan to repeat this educational program by adding larger population in a wider community scale annually.

References:

1-Salti etal, Diabetes Care 2004; 27: 2306

2-Diabetes care, volume 33, number 8,2010 ; IDF congress 2013, abstract no:ME-1555

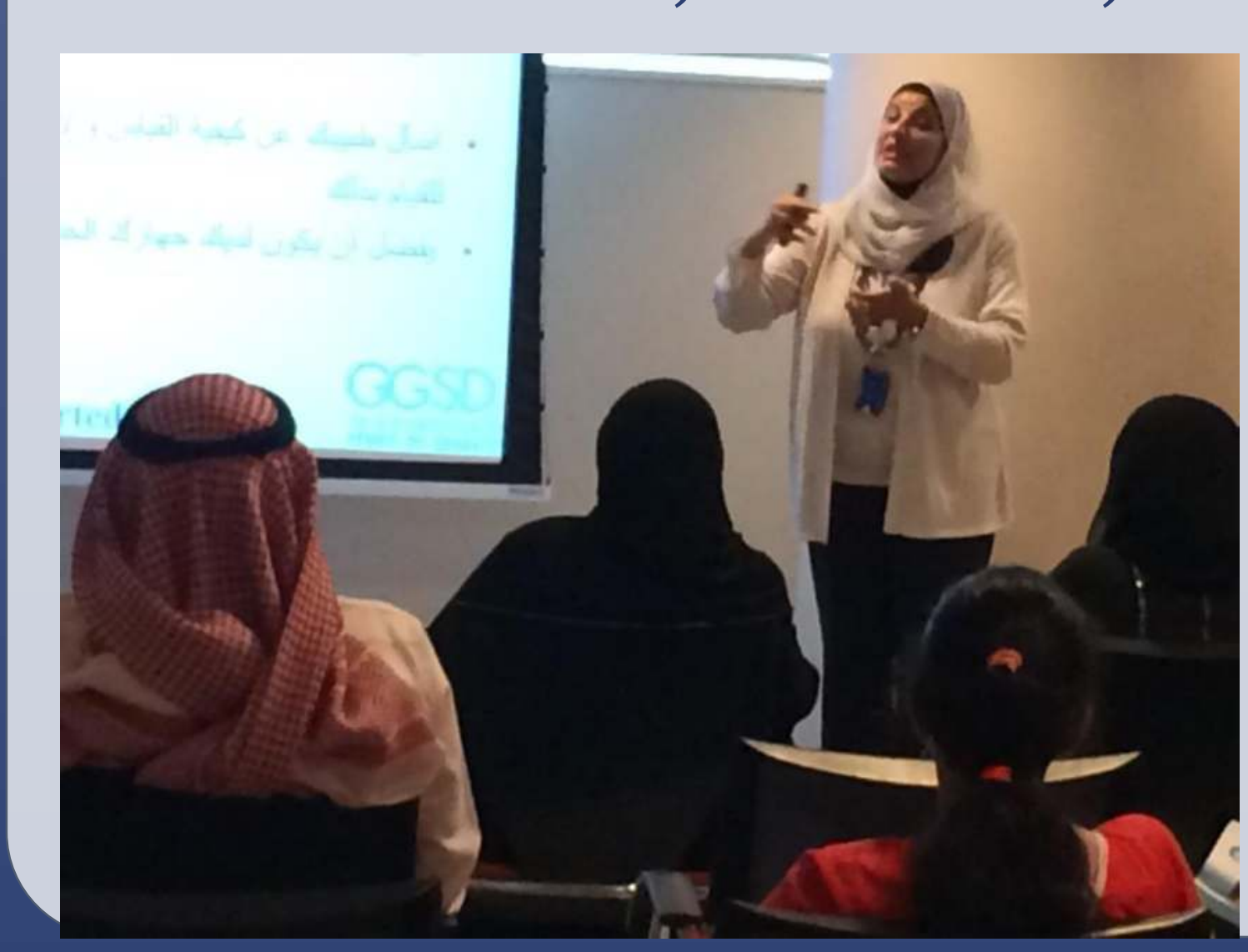

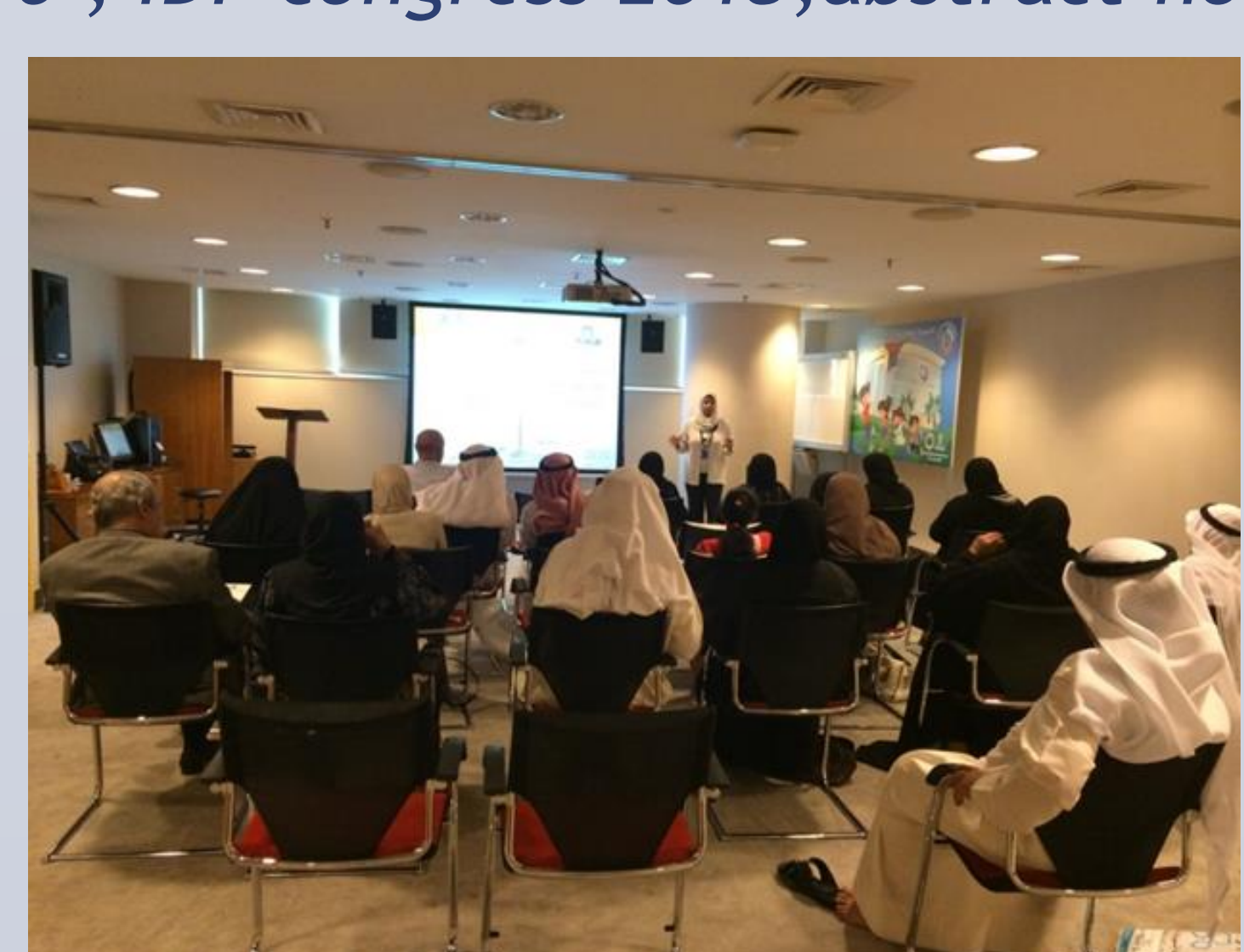

